\title{
Broadband matched-field processing: Coherent and incoherent approaches
}

\author{
Cristiano Soares $^{\mathrm{a})}$ and Sérgio M. Jesus ${ }^{\mathrm{b})}$ \\ SiPLAB-FCT, Universidade do Algarve, Campus de Gambelas, 8000-Faro, Portugal
}

(Received 2 January 2002; revised 7 October 2002; accepted 30 January 2003)

\begin{abstract}
Matched-field based methods always involve the comparison of the output of a physical model and the actual data. The method of comparison and the nature of the data varies according to the problem at hand, but the result becomes always largely conditioned by the accurateness of the physical model and the amount of data available. The usage of broadband methods has become a widely used approach to increase the amount of data and to stabilize the estimation process. Due to the difficulties to accurately predict the phase of the acoustic field the problem whether the information should be coherently or incoherently combined across frequency has been an open debate in the last years. This paper provides a data consistent model for the observed signal, formed by a deterministic channel structure multiplied by a perturbation random factor plus noise. The cross-frequency channel structure and the decorrelation of the perturbation random factor are shown to be the main causes of processor performance degradation. Different Bartlett processors, such as the incoherent processor [Baggeroer et al., J. Acoust. Soc. Am. 80, 571-587 (1988)], the coherent normalized processor [Z.-H. Michalopoulou, IEEE J. Ocean Eng. 21, 384-392 (1996)] and the matched-phase processor [Orris et al., J. Acoust. Soc. Am. 107, 2563-2375 (2000)], are reviewed and compared to the proposed cross-frequency incoherent processor. It is analytically shown that the proposed processor has the same performance as the matched-phase processor at the maximum of the ambiguity surface, without the need for estimating the phase terms and thus having an extremely low computational cost. (C) 2003 Acoustical Society of America. [DOI: 10.1121/1.1564016]
\end{abstract}

PACS numbers: 43.30.Wi, 43.30.Pc, 43.60.Cg [DLB]

\section{INTRODUCTION}

The introduction of physical models in underwater acoustic signal processing has been one of the most significant advances ever in this field. ${ }^{1-3}$ Defining a physical model for a given practical scenario allows for a consistent inclusion of a priori information on the signal estimation processor. That a priori information consists of the environmental characteristics of the propagation scenario which, by means of the solution of the wave equation on that scenario, restricts the received acoustic pressure to a well-defined class of expected signals. It is that reduction of the class of expected signals that provides the highest performance gain in terms of parameter estimation.

Since the definition of a physical model requires the knowledge (or the assumption) of a number of environmentally measurable quantities, the performance of the processor becomes dependent on those quantities. Conversely, if the emitted and received signals are known (or measurable) then it is, in principle, possible to estimate the environmental characteristics of the media of propagation - that is the base of the various matched-field (MF) based techniques being developed in the last two decades: Matched-field processing (MFP) for source localization, matched-field tomography (MFT) for ocean properties and matched-field inversion (MFI) for geoacoustic parameter estimation.

There are at least two aspects that emerge by their re-

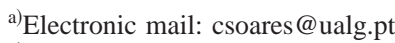

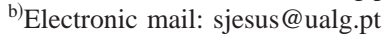

levance to the success of MF based techniques: one is the ability of a given MF processor to accurately pinpoint the source location while rejecting sidelobes, and the other is the impact of erroneous or missing environmental information (known as model mismatch) in the final parameter estimate. This study addresses the first aspect, regarding sidelobe rejection, while considering that the processor is working on a mismatch free situation. In that case, the capacity of detecting the correct acoustic field among very close similar candidates (the so-called discrimination) largely depends on the degree of complexity of the received acoustic pressure field. As an example, a single tone will have two discriminating parameters: the amplitude and the phase. If a broadband signal is transmitted, there are as many amplitudes and phases as discrete frequencies, and the complexity of the received signal is naturally increased leading to a higher MF discrimination. This problem is similar-but not equal-to the detection problem encountered in classical spectrum estimation.

There are a number of different ways to combine MF information across frequency that can be classified in two broad groups: the conventional incoherent methods, that are based on the direct averaging of the autofrequency inner products (average of real numbers) and the, say, less conventional methods, that perform a weighted average of the crossfrequency inner products where the weights are the frequency compensated phase shifts. The latter are generally called coherent broadband methods since they combine complex inner products.

Incoherent MF methods were first proposed by Baggeroer et al. ${ }^{4}$ where geometric averaging was found to be ef- 
fective to reduce ambiguous Bartlett and minimum variance (MV) MFP sidelobes in a shallow-water simulation study. The same principle was used in a countless number of studies since then. More recently, the frequency domain coherent approach was first suggested by Tolstoy. ${ }^{5}$ Michalopoulou recognized that incoherent processors discarded useful information contained in the off-diagonal terms of the crossfrequency data covariance matrix. ${ }^{6}$ Coherent Bartlett and MV processors based on the formulation of "supervectors" containing field vectors of the frequencies to be processed were proposed and successfully applied on tracking a sound source in the Hudson Canyon data set. ${ }^{7}$ Czenszak and Krolik proposed a coherent minimum variance beamformer with environmental perturbation constrains (MV-EPC) designed for a short vertical array. ${ }^{8}$ Very recently Orris et al. proposed a matched-phase coherent processor that accounts for the relative phase relationships between frequencies. ${ }^{9}$ Those phases are assumed to be unknown and are searched as free parameters.

In that classification, time domain methods play a different role but can, to some extent, be included in the coherent class. Time domain methods were first suggested by Clay ${ }^{10}$ under the form of an optimum matched-filter for source localization. The same technique was used by Li et al. ${ }^{11}$ in laboratory experimental data. Also Frazer et al. ${ }^{12}$ tested Clay's technique with simulated data and a single hydrophone. In 1992, Miller et al. ${ }^{13}$ showed, with computer simulations, that it is possible to localize short duration acoustic signals in a range-dependent shallow water environment. The same approach was followed by Knobles et al. ${ }^{14}$ with bottom moored sensors using a broadband coherent matched-field processor proposed by Westwood. ${ }^{15}$ Time domain source localization was actually achieved with real data by Brienzo et al. ${ }^{16}$ using data received on a vertical array in a deep water area on the Monterrey fan. The technique used was a combination of time domain filtering for each sensor (matchedfilter) and then a space domain beamformer.

Despite the considerable amount of work on broadband methods there is a lack of understanding on why and when a coherent method provides a better detection or localization performance than an incoherent method. This is the main topic addressed in the present study, that starts by presenting a physical-based linear data model with suitable random perturbation terms as opposed to the traditional fully stochastic model. Under this model, it is shown that the advantage of using the cross-frequency terms resides in its ability to reject noise, while its disadvantage is that the result is limited by the correlation of the random phase terms together with the deterministic correlation of the channel response across frequency. An efficient algorithm for combining crossfrequency information is derived that is shown to have an equivalent localization performance than that of the matched-phase coherent processor with a much lower computational burden. Then, the performance of the coherent and incoherent processors are compared for different number of frequencies using simulated data. Real data analysis is presented to support the physical-based model as well as for justifying the distributions of the random perturbation terms. Finally, a real data example shows the effect of a wise selec- tion of frequency bands on the final match of the model to the data.

\section{DATA MODEL}

\section{A. The physical data model}

A widely used data model for $M$ farfield point sources emitting narrowband signals received in a L-sensor array is given by

$$
\mathbf{y}(t)=\mathbf{A}(\varphi) \mathbf{s}(t)+\mathbf{u}(t),
$$

where $\mathbf{y}(t)$ is the L-sensor array received acoustic pressure, $\mathbf{A}(\varphi)$ is the $L \times M$ steering matrix, which entries are the appropriate delays for each array sensor and each source $m$ at bearing $\varphi_{m}, \mathbf{s}(t)$ is an $M$-dimensional vector with the $M$ source inputs at time $t$ and $\mathbf{u}(t)$ is the observation additive noise. A common assumption is to consider that the additive noise is white, Gaussian, zero-mean and uncorrelated with the signals $\mathbf{s}(t)$, that themselves are zero-mean and uncorrelated stochastic processes. This model is useful for describing a field of dependent noise sources emitting through a nondispersive unbounded media and received on a horizontal array. When dealing with shallow water dispersive scenarios, deterministic sources and nonhorizontal arrays this model is unable to account for the complexity of the received field as a mixture of correlated (partially) deterministic signal reflections from sea bottom and sea surface.

An alternative approach is to start from the wave equation and directly calculate its solution with appropriate boundary conditions and environmental assumptions (e.g., azimuth and range independent isotropic media, spatial point source, etc.). In a cylindrical two-dimensional coordinate system, the acoustic pressure measured at receiver depth $z_{l}$ due to a point source at range $r$ and depth $z_{s}$ can be written ${ }^{17}$

$$
\begin{aligned}
p\left(z_{l}, t ; r, z_{s}\right)= & \frac{-i}{2 \pi} \int \sum_{j=1}^{J_{M}} s(\omega) \frac{\Psi_{j}\left(z_{l}\right) \Psi_{j}\left(z_{s}\right)}{\sqrt{r k_{j}}} \\
& \times e^{-i\left(k_{j} r-\pi / 4\right)-\gamma_{j} r} e^{i \omega t} d \omega,
\end{aligned}
$$

where $s(\omega)$ is the source spectrum, $\left\{\Psi_{j}(), j=1, \ldots, J_{M}\right\}$ are the waveguide mode functions, and $k_{j}$ and $\gamma_{j}$ are the mode horizontal wave numbers and mode attenuation coefficients, respectively; $J_{M}$ is the number of discrete modes supported by the waveguide.

Under the ray approximation the received acoustic pressure, using the same notation, can be written as

$p\left(z_{l}, t ; r, z_{s}\right)=\frac{1}{2 \pi} \int \sum_{j=1}^{J_{R}} a_{j} s(\omega) e^{-i \omega \tau_{j}} e^{i \omega(t+|\pi / 2 \omega|)} d \omega$,

where the number of eigenrays $J_{R}$, the ray amplitudes $a_{j}$ and the delays $\tau_{j}$, fully characterize the propagation channel for the specific source and receiver locations, $\left(0, z_{s}\right)$ and $\left(r, z_{l}\right)$, respectively.

Assuming the propagation channel as a linear filter, allows for writing the received signal as the frequency product between the source signal $s(\omega)$ and the channel transfer function $h(\omega)$, defined as the sum of modal terms (or rays) 
for a particular source-receiver location. Thus, a suitable model for the array-received signal from an harmonic source at frequency $\omega$ would be

$$
y\left(z_{l}, \omega ; r, z_{s}\right)=h\left(z_{l}, \omega ; r, z_{s}\right) s(\omega)+u\left(z_{l}, \omega\right),
$$

where $u\left(z_{l}, \omega\right)$ is a zero-mean stochastic process representing additive observation noise and where $h\left(z_{l}, \omega ; r, z_{s}\right)$ can be easily deduced either from (2) or from (3) depending on which model-normal mode or ray model-is being used. It is a common assumption to consider the observation noise to be wide-sense time stationary. Taking into account the Fourier transform properties for sufficiently long observation times it can be considered that the frequency samples of $u$ are asymptotically uncorrelated.

If the source input $s(t)$ is deterministic, signal detection using model (4) becomes a problem of detecting a deterministic signal in white noise, which optimal solutions are well known.

In the past decade, with the development of methods for acoustic inversion using deterministic signals, it has been observed that repeated emissions at very high SNR resulted in successive receptions suffering rapid changes in short time intervals possibly caused by small scale environmental perturbations, source and/or receiver motion, and sea surface and bottom roughness, which, partially or all together, contribute to unmodeled fluctuations in the signal part of (4).

Since such changes cannot be attributed to the noise due to the high SNR, a complex random factor $\alpha=|\alpha| \exp (j \phi)$ can be included such that the data model is written as

$$
\mathbf{y}\left(\omega, \theta_{0}\right)=\alpha(\omega) \mathbf{h}\left(\omega, \theta_{0}\right) s(\omega)+\mathbf{u}(\omega),
$$

where a more compact notation has been adopted by introducing a vectorial notation for the L-sensor array as $\mathbf{y}$ $=\left[y\left(z_{1}\right), y\left(z_{2}\right), \ldots, y\left(z_{L}\right)\right]^{t}$ and similar definitions for $\mathbf{h}$ and $\mathbf{u}$, the channel transfer function and the additive observation noise, respectively; $s(\omega)$ is the source spectrum at frequency $\omega$ and $\theta_{0}$ is a vector with the relevant parameters under estimation. The noise process $\mathbf{u}$ is assumed to be uncorrelated from sensor to sensor and with random factor $\alpha$. Note that random factor $\alpha$ is space invariant but is assumed to be frequency dependent. For the design of optimal estimators it is useful to consider that $\alpha$ is zero-mean and Gaussian distributed. Whether that assumption is verified in practice is the subject of the next section.

\section{B. Random signal perturbation factor}

This section deals with the distribution of the random signal perturbation factor $\alpha$, introduced in the linear physical model (5). It is a common assumption to consider that random factor to be complex zero-mean Gaussian distributed, ${ }^{4}$ which implies that the module of $\alpha$ follows a Rayleigh distribution and that its phase is uniformly distributed in $[-\pi$, $\pi] .{ }^{18}$ In case that the real and/or imaginary parts of the acoustic pressure are not zero mean then the envelope follows a Rice distribution while the phase term does not appear to be uniform nor Gaussian distributed (see Appendix A).

In order to obtain an empirical distribution of the signal random perturbation, only possible using real data, one has to, first, assume that the signal-to-noise ratio (SNR) is sufficiently high, to be able to neglect the influence of the noise $\mathbf{u}$, and second, assume that the deterministic part of the signal, i.e., $\mathbf{h}\left(\omega, \theta_{0}\right) s(\omega)$ is time-stationary or slowly varying. Under these two assumptions a possible estimator, $\hat{\alpha}$, of the random factor $\alpha$ at frequency $\omega$ is

$$
\hat{\alpha}_{n}=\frac{y_{n}}{y_{0}} \approx \frac{\left|\alpha_{n}\right|}{\left|\alpha_{0}\right|} e^{j\left(\phi_{n}-\phi_{0}\right)},
$$

where $y_{n}, \alpha_{n}$, and $\phi_{n}$ are obtained for time snapshot $n$ and for an arbitrary frequency and receiver. This would imply that the distribution of $|\hat{\alpha}|$ would be Rayleigh or Rice depending on whether $\alpha$ is zero-mean or not with, however, a change on the amplitude axis due to the normalization constant $\left|\alpha_{0}\right|$. As an alternative and, if the stationarity assumption for $\mathbf{h}\left(\omega, \theta_{0}\right) s(\omega)$ is suspected not to hold, another estimator can be sought using a time sliding estimator as

$$
\hat{\alpha}_{n}=\frac{y_{n}}{y_{n-1}} \approx \frac{\left|\alpha_{n}\right|}{\left|\alpha_{n-1}\right|} e^{j\left(\phi_{n}-\phi_{n-1}\right)} .
$$

In this case the interpretation is a bit more elaborated since the module of $\alpha$ is the ratio of two Rayleigh (or Rice) random variables and the phase term is the difference between two uniform variables if $\alpha$ is zero mean. It is shown in Appendix B that the ratio of two independent Rayleigh distributed random variables gives a nearly Cauchy distributed random variable and that the difference of two uniformly distributed and independent random variables gives a probability density function (pdf) for the resulting random variable that is triangular in $[-2 \pi, 2 \pi]$. Results obtained on real data using estimators (6) and (7) are shown in Sec. VI.

\section{SECOND ORDER STATISTICS AND BROADBAND MODEL FORMULATION}

The correlation matrix can be directly written from (5) as

$$
\begin{aligned}
\mathbf{C}_{y y}\left(\omega, \theta_{0}\right)= & E\left[\mathbf{y}\left(\omega, \theta_{0}\right) \mathbf{y}^{H}\left(\omega, \theta_{0}\right)\right] \\
= & E\left[|\alpha(\omega)|^{2}\right]|s(\omega)|^{2} \mathbf{h}\left(\omega, \theta_{0}\right) \mathbf{h}^{H}\left(\omega, \theta_{0}\right) \\
& +\sigma_{u}^{2}(\omega) \mathbf{I},
\end{aligned}
$$

where all terms have been previously defined and superscript $H$ denotes conjugate transpose. Equation (5) gives the essential description of the received data model in the narrowband case. When a time-limited signal (impulse) is emitted by the source, a significant band of frequencies of the acoustic channel is excited giving rise to the need for a broadband formulation. In order to introduce, as much as possible, a common frame for the narrowband and broadband cases, we define an extended vector as

$$
\underline{\mathbf{y}}=\left[\mathbf{y}^{T}\left(\omega_{1}\right), \mathbf{y}^{T}\left(\omega_{2}\right), \ldots, \mathbf{y}^{T}\left(\omega_{K}\right)\right]^{T},
$$

where superscript $T$ denotes matrix transpose and $K$ is the total number of discrete frequency bins. In that case, the broadband model can be written as

$$
\underline{\mathbf{y}}\left(\theta_{0}\right)=\mathbf{H}\left(\theta_{0}\right) \underline{\widetilde{\mathbf{s}}}+\underline{\mathbf{u}},
$$


where $\underline{\mathbf{s}}$ is a $K$-dimensional random vector which entries are $s\left(\omega_{k}\right) \alpha\left(\omega_{k}\right)$, i.e., the source spectrum multiplied by the random perturbation factor at each frequency $\omega_{k} \in\left[\omega_{1}, \omega_{K}\right]$; the matrix $\mathbf{H}\left(\theta_{0}\right)$ is

$$
\mathbf{H}\left(\theta_{0}\right)=\left[\begin{array}{cccc}
\mathbf{h}\left(\omega_{1}, \theta_{0}\right) & 0 & \cdots & 0 \\
0 & \mathbf{h}\left(\omega_{2}, \theta_{0}\right) & \cdots & 0 \\
\vdots & \vdots & \ddots & \vdots \\
0 & 0 & \cdots & \mathbf{h}\left(\omega_{K}, \theta_{0}\right)
\end{array}\right],
$$

where the noise extended vector $\underline{\mathbf{u}}$ has an obvious notation similar to (9). It is interesting to write the correlation matrix for model (10), which cross-frequency block matrix is given by

$$
\begin{aligned}
& \mathbf{C}_{y y}\left(\omega_{i}, \omega_{j}\right) \\
& =\left\{\begin{array}{l}
\left|s\left(\omega_{i}\right)\right|^{2} \mathbf{h}\left(\omega_{i}, \theta_{0}\right) \mathbf{h}\left(\omega_{i}, \theta_{0}\right)^{H} E\left[\left|\alpha\left(\omega_{i}\right)\right|^{2}\right]+\sigma_{u}^{2}\left(\omega_{i}\right) \mathbf{I}, \\
\quad i=j, \\
s\left(\omega_{i}\right) s^{*}\left(\omega_{j}\right) \mathbf{h}\left(\omega_{i}, \theta_{0}\right) \mathbf{h}^{H}\left(\omega_{j}, \theta_{0}\right) E\left[\alpha\left(\omega_{i}\right) \alpha^{*}\left(\omega_{j}\right)\right], \\
i \neq j,
\end{array}\right.
\end{aligned}
$$

where the term $E\left[\alpha\left(\omega_{i}\right) \alpha^{*}\left(\omega_{j}\right)\right]$ denotes the correlation of the perturbation factor across frequency. Note that unlike the autofrequency entries $(i=j)$ the cross-frequency terms $(i$ $\neq j$ ) are noise free. This is due to the well-known property of the Fourier transform for time-stationary processes that gives uncorrelated cross-frequency bins which might be also useful if spatially correlated noise is present. In practice, with finite observation time, that property is only asymptotically verified, which is often sufficient. In expression (12), for $i \neq j$, there are three contributions: the source cross-spectrum term $s\left(\omega_{i}\right) s^{*}\left(\omega_{j}\right)$, the cross-frequency acoustic channel structure term $\mathbf{h}\left(\omega_{i}, \theta_{0}\right) \mathbf{h}^{H}\left(\omega_{j}, \theta_{0}\right)$ and the perturbation factor correlation $E\left[\alpha\left(\omega_{i}\right) \alpha^{*}\left(\omega_{j}\right)\right]$. The first term is source dependent and will not be of concern here. The second term is channel dependent and may significantly vary with environmental conditions, source position (range and depth) and receiving array geometry. The third term on expression (12), for $i$ $\neq j$, concerns the correlation of the perturbation factor and is impossible to obtain from simulations.

\section{BARTLETT MATCHED-FIELD PROCESSING}

The Bartlett processor is possibly the most widely used estimator in MF parameter identification. The parameter estimate $\hat{\theta}_{0}$ is given as the argument of the maximum of the functional

$$
P(\theta)=E\left[\hat{\mathbf{w}}^{H}(\theta) \mathbf{y}\left(\theta_{0}\right) \mathbf{y}^{H}\left(\theta_{0}\right) \hat{\mathbf{w}}(\theta)\right],
$$

where the replica vector estimator is determined as the vector $\mathbf{w}(\theta)$ that maximizes the mean quadratic power,

$$
\hat{\mathbf{w}}(\theta)=\arg \max _{\mathbf{w}} E\left[\mathbf{w}^{H}(\theta) \mathbf{y}\left(\theta_{0}\right) \mathbf{y}^{H}\left(\theta_{0}\right) \mathbf{w}(\theta)\right],
$$

subject to $\mathbf{w}^{H}(\theta) \mathbf{w}(\theta)=1$. In the narrowband case, using model (5) in (14) gives the well-known nontrivial solution

$$
\hat{\mathbf{w}}_{N B}(\theta)=\frac{\mathbf{h}(\theta)}{\sqrt{\mathbf{h}^{H}(\theta) \mathbf{h}(\theta)}},
$$

where the denominator is a normalization scalar and the numerator contains the signal structure as "seen" at the receiving array. This is simply the classical matched filter for the particular parameter location $\theta$. Substituting (15) in (13) gives the well-known generalized conventional narrow band beamformer for parameter $\theta$. If the search is made over $\theta$ and the maximum is selected, then an optimum mean leastsquares estimate $\hat{\theta}_{0}$ of $\theta_{0}$ is obtained.

In the broadband case, the estimator of the replica vector is given in terms of frequency extended vectors using model (10), thus

$$
\hat{\mathbf{w}}_{B B}(\theta)=\arg \max _{\underline{\mathbf{w}}}\left\{\underline{\mathbf{w}}^{H}(\theta) \mathbf{H}\left(\theta_{0}\right) E\left[\underline{\widetilde{\mathbf{s}}}^{H}\right] \mathbf{H}^{H}\left(\theta_{0}\right) \underline{\mathbf{w}}(\theta)\right\},
$$

where the expectation of the signal matrix $\underline{\widetilde{S}}^{H}$ relates to the correlation of the perturbation factor $\alpha$ across frequency, weighted by the source power cross spectrum $s^{*}\left(\omega_{i}\right) s\left(\omega_{j}\right)$. No closed form for $\hat{\underline{\underline{w}}}_{B B}(\theta)$ can be given in this case without explicit knowledge of that signal matrix. There are a number of possible implementations that represent suboptimal versions of (16) with different assumptions for the structure of the perturbation correlation and signal weighting matrix. A few cases are reviewed in the next section and a new computational effective alternative to existing techniques is also proposed.

\section{A. Broadband incoherent processor}

The so-called incoherent broadband Bartlett processor, originally proposed in Ref. 4, implicitly assumes that the random factor is simply $E\left[\alpha\left(\omega_{i}\right) \alpha^{*}\left(\omega_{j}\right)\right]=\sigma_{\alpha}^{2} \delta_{i j}$, i.e., that the random perturbations are uncorrelated across frequency and have a constant power. Using that expression of the correlation of $\alpha$ in (12), plugged in (16) and solved for $\mathbf{w}$ gives

$$
\underline{\hat{\mathbf{w}}}_{\mathrm{inc}}(\theta)=\frac{\mathbf{H}(\theta) \underline{\mathbf{s}}}{\|\mathbf{H}(\theta) \underline{\mathbf{s}}\|},
$$

where $\underline{\mathbf{s}}$ is a $K$-dimensional vector which entries are $s\left(\omega_{k}\right)$. Thus, by replacement into (13), allows to obtain the processor expression

$P_{\text {inc }}(\theta)=\frac{\sigma_{\alpha}^{2} \sum_{k=1}^{K}\left|s\left(\omega_{k}\right)\right|^{2} \mathbf{h}^{H}\left(\omega_{k}, \theta\right) \mathbf{C}_{y y}\left(\omega_{k}, \omega_{k}\right) \mathbf{h}\left(\omega_{k}, \theta\right)}{\|\mathbf{H}(\theta) \underline{\mathbf{s}}\|^{2}}$

which is nothing more than a source power weighted sum of the diagonal matched-filtered autofrequency block matrices of the extended correlation matrix $\mathbf{C}_{y y}$. Notice that if $\sigma_{\alpha}$ had been assumed to be frequency dependent, a factor $\sigma_{\alpha}\left(\omega_{k}\right)$ would appear as weighting the terms in the summation in (18). In the case of a flat source power spectrum, Eq. (18) reduces to a simple summation of the quadratic terms across the discrete band of frequencies. When the source power spectrum is unknown but not flat, an unweighted incoherent processor is generally used which leads to the suboptimal incoherent broadband conventional estimator proposed in Ref. 4. 


\section{B. Broadband coherent processor}

Although there is good evidence that for many of the real underwater propagation channels most of the energy is concentrated along the main diagonal of the cross-spectrum correlation matrix (the autofrequency terms) it is also clear that the same autofrequency terms would carry the noise power as it can be seen in expression (12). One of the motivations when performing coherent processing is to take advantage of the noiseless cross-frequency terms of (12). These cross-frequency terms have no noise but the signal information they contain may also be reduced, according to both the channel cross-frequency structure and the cross-frequency correlation of the random perturbation factor, as explained in the preceding section. This explains why in most studies, concerned with coherent processing, only the crossfrequency off-diagonal terms were used, excluding the diagonal autofrequency information. ${ }^{9}$ There are actually several broadband coherent processors depending on the assumptions made for approximating the cross-frequency perturbation terms of the signal matrix $E\left[\widetilde{\mathbf{S}}^{H}\right]$ of (16).

\section{Coherent normalized processor}

The coherent normalized processor $(\mathrm{COH}-\mathrm{N})$ has been proposed by Michalopoulou, ${ }^{7,19}$ and attempts to eliminate the source spectrum-perturbation weighting across frequency. At each frequency $\omega_{i}$, a normalized model vector is defined as

$$
\mathbf{n}_{x}\left(\omega_{i}, \theta_{0}\right)=\frac{\mathbf{x}\left(\omega_{i}, \theta_{0}\right)}{x_{l}\left(\omega_{i}, \theta_{0}\right)},
$$

where $x_{l}\left(\omega_{i}, \theta_{0}\right)$ is the signal received at sensor $l$. The choice of $l$ depends on the actual signal-to-noise ratio (SNR) at that particular sensor. In a high SNR situation, if the noise contribution at sensor $l$ is neglected, the normalized data model becomes

$$
\mathbf{n}_{x}\left(\omega_{i}, \theta\right) \approx \mathbf{n}_{h}\left(\omega_{i}, \theta_{0}\right)+\frac{\mathbf{u}\left(\omega_{i}\right)}{h_{l}\left(\omega_{i}, \theta_{0}\right) s\left(\omega_{i}\right) \alpha\left(\omega_{i}\right)} .
$$

Matching this model with an extended normalized replica vector yields a perfect match for the signal and a strongly correlated structure for the noise field due to the noise term in (20). In that case the coherent-normalized replica vector is written as

$$
\hat{\mathbf{w}}_{\mathrm{coh}-n}\left(\omega_{i}, \theta\right)=\mathbf{n}_{h}\left(\omega_{i}, \theta\right)=\frac{\mathbf{h}\left(\omega_{i}, \theta\right)}{h_{l}\left(\omega_{i}, \theta\right)},
$$

and using that expression in the Bartlett processor gives

$$
\begin{aligned}
& P_{\text {coh }-n}(\theta) \\
& =\sum_{i=1}^{K} \sum_{j=1}^{K} \mathbf{n}_{h}^{H}\left(\omega_{i}, \theta\right) \mathbf{n}_{h}\left(\omega_{i}, \theta_{0}\right) \mathbf{n}_{h}^{H}\left(\omega_{j}, \theta_{0}\right) \mathbf{n}_{h}\left(\omega_{j}, \theta\right) \\
& \quad+\mathbf{n}_{h}^{H}\left(\omega_{i}, \theta\right) \mathbf{C}_{n_{u} n_{u}}\left(\omega_{i}, \omega_{j}\right) \mathbf{n}_{h}\left(\omega_{j}, \theta\right),
\end{aligned}
$$

where $\mathbf{C}_{n_{u} n_{u}}\left(\omega_{i}, \omega_{j}\right)$ is the cross-frequency correlation matrix of the normalized additive noise vector $\mathbf{n}_{u}$ defined in the second term of (20). Expression (22) shows a perfectly coherent match for the signal model part when $\theta=\theta_{0}$, and a noise term residual which is a constant when $i=j$, due to the white noise assumption, and has a correlation structure for $i \neq j$ that is highly dependent on the cross-frequency correlation of the perturbation $\alpha(\omega)$.

\section{Matched-phase coherent processor}

Another approximation to the broadband coherent processor has been recently proposed by Orris $^{9}$ where the correlation terms are explicitly included in the replica vector as unknowns and have therefore to be estimated. A new replica vector is defined as

$\underline{\mathbf{w}}_{\text {coh-mp }}(\theta)=\left[\mathbf{h}^{T}\left(\omega_{1}, \theta\right) e^{j \hat{\phi}_{h}\left(\omega_{1}\right)}, \ldots, \mathbf{h}^{T}\left(\omega_{K}, \theta\right) e^{j \hat{\phi}_{h}\left(\omega_{K}\right)}\right]^{T}$,

where the phase terms $\left[\hat{\phi}_{h}\left(\omega_{k}\right) ; k=1, \ldots, K\right]$ are the estimates that maximize the output power upon summation over sensor and frequency. Taking into account that, when carrying out that summation, each term has its complex conjugate, the energy contained in the imaginary part is lost. The unknown phase terms $\phi_{h}$ are estimated in such a way as to minimize that loss which, ideally, requires the unknown phase terms to be symmetric to the phase of the signal matrix terms in (12). If that is achieved all terms turn into real numbers and the sum is carried out in phase. In that case, and for a flat spectrum source, this processor is optimum. Replacing (23) in the Bartlett processor expression gives

$$
\begin{aligned}
P_{\text {coh-mp }}(\theta)= & \sum_{i=1}^{K} \sum_{l=1}^{K} \mathbf{h}^{H}\left(\omega_{i}, \theta\right) \mathbf{C}_{y y}\left(\omega_{i}, \omega_{l}\right) \\
& \times \mathbf{h}\left(\omega_{l}, \theta\right) e^{-j\left[\hat{\phi}_{h}\left(\omega_{i}\right)-\hat{\phi}_{h}\left(\omega_{l}\right)\right]} .
\end{aligned}
$$

In practice, the problem associated to the matched-phase processor, according to Orris, ${ }^{9}$ is the computation load necessary to obtain the estimates $\hat{\phi}_{h}$ of the phase shifts $\phi_{h}$, for an exhaustive search over a realistic parameter space. That computation load is of the order of $o=J^{K} \times M \times N$, where $J$ is the number of samples for the phase in $[0,2 \pi], K$ is the number of frequencies and $M \times N$ is the $\theta$ parameter search grid (e.g., range versus depth). In practice, and as mentioned by Orris, ${ }^{9}$ if the source location and relative phases have to be exhaustively searched, computation complexity limits the number of frequencies to $K=3$ while for a larger number of frequencies efficient search algorithms (e.g., simulated annealing) were proposed.

\section{The cross-frequency incoherent processor}

The cross-frequency incoherent processor is proposed in this paper and represents an alternative to overcome the computational burden of the matched-phase processor while keeping the same performance. This processor stems from the simple idea that the phase corrections for the surface maximum $\left(\theta=\theta_{0}\right)$ are

$$
\phi_{h}\left(\omega_{i}\right)-\phi_{h}\left(\omega_{j}\right)=\angle s\left(\omega_{i}\right) s^{*}\left(\omega_{j}\right) E\left[\alpha\left(\omega_{i}\right) \alpha^{*}\left(\omega_{j}\right)\right] \text {, }
$$

for all $i, j=1, \ldots, K$ which can be seen by direct inspection of (12) and where $\angle$ means "phase of." When these corrections are correctly set the value of the maximum is just the sum of a series of real numbers, which are the modules of the quadratic terms across frequency, i.e., 
(a)

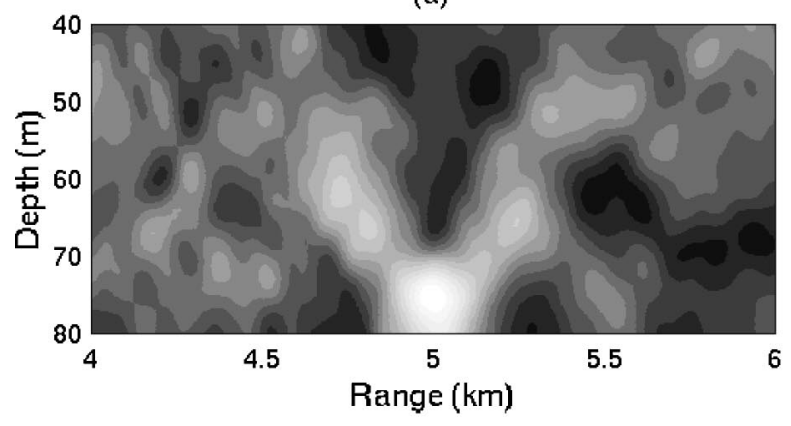

(c)

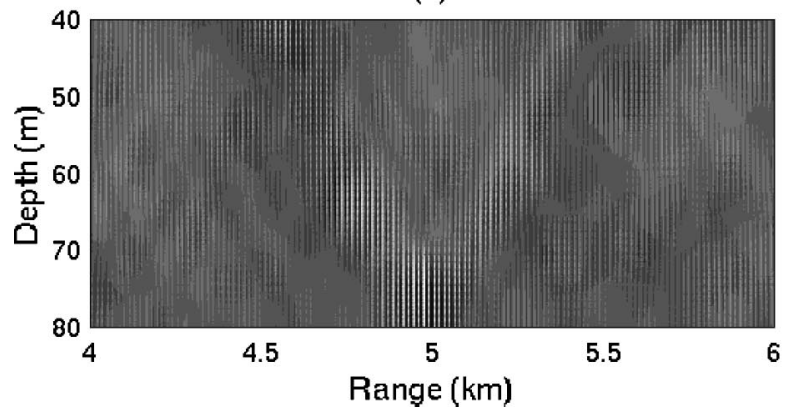

(b)

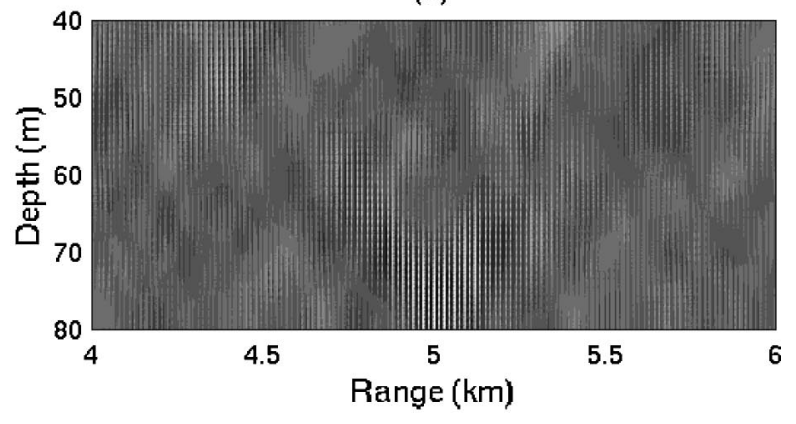

(d)

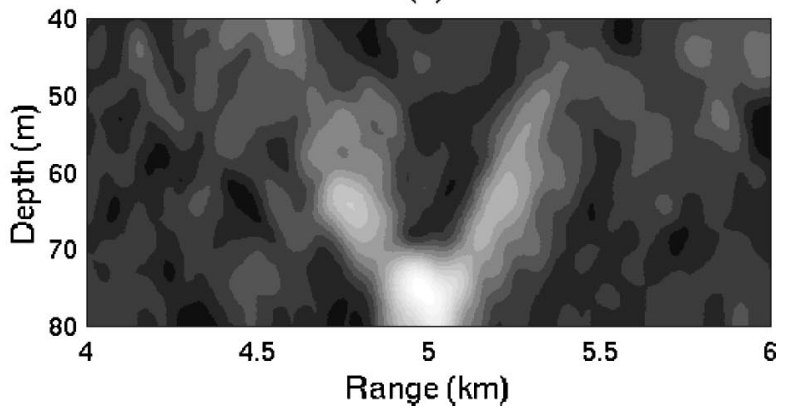

FIG. 1. Ambiguity surfaces computed with synthetic data generated without perturbation factor for the ADVENT'99 scenario at frequencies 300 , 400 , 500 , and $600 \mathrm{~Hz}$, at SNR $=-8 \mathrm{~dB}$ and for the following processors: (a) incoherent conventional, (b) coherent normalized, (c) matched-phase coherent, and (d) incoherent cross frequency.

$$
P_{\text {inc-xf }}(\theta)=\sum_{i=1}^{K} \sum_{j=1}^{K}\left|\mathbf{h}^{H}\left(\omega_{i}, \theta\right) \mathbf{C}_{y y}\left(\omega_{i}, \omega_{j}\right) \mathbf{h}\left(\omega_{j}, \theta\right)\right| .
$$

The value of the maximum of the ambiguity surface obtained with (26) is exactly the same as that obtained with (24) with absolutely no phase parameter search. Therefore, the peak would have the same height and the same location, however the aspect of the resulting surface would be much different between the cross-frequency and the matched-phase processors: the former would have a smooth appearance, much like the incoherent processor, and the latter would have extremely narrow peaks distributed along the surface with, however, an overall envelop that is very similar to that of the crossfrequency incoherent processor. Examples on simulated data are given in the next section.

\section{SIMULATION RESULTS}

This section shows a few simulated data examples of the application of broadband MF processors to source localization. The data was simulated using the C-SNAP model ${ }^{20}$ in a $80 \mathrm{~m}$ deep range-independent shallow water scenario similar to that of the ADVENT'99 experiment. ${ }^{21}$ The acoustic source is placed at $76 \mathrm{~m}$ depth and at $5 \mathrm{~km}$ range from a 32 -sensors vertical array. The source is emitting a series of multitones between 300 and $600 \mathrm{~Hz}$ with $100 \mathrm{~Hz}$ increment. The signals were generated in the frequency domain using (4) with an SNR of $-8 \mathrm{~dB}$ and the correlation matrix was estimated using 32 snapshots. The noise level was set accordingly to the following SNR definition:

$$
\mathrm{SNR}_{\mathrm{dB}}=10 \log \frac{\sum_{k=1}^{K} \sigma_{s}^{2}\left(\omega_{k}\right)}{\sum_{k=1}^{K} \sigma_{u}^{2}\left(\omega_{k}\right)},
$$

where

$$
\sigma_{s}^{2}\left(\omega_{k}\right)=\frac{E\left[\left\|\mathbf{h}\left(z_{l}, \omega_{k}, r, z_{s}\right) s\left(\omega_{k}\right)\right\|^{2}\right]}{L}
$$

and

$$
\sigma_{u}^{2}\left(\omega_{k}\right)=\frac{E\left[\left\|\mathbf{u}\left(\omega_{k}\right)\right\|^{2}\right]}{L} .
$$

Figure 1 shows the range-depth ambiguity surfaces obtained for the above referred broadband Bartlett processors, $P_{\text {inc }}$ (a), $P_{\text {coh- } n}$ (b), $P_{\text {coh-mp }}\left(\right.$ c), and $P_{\text {inc-xf }}(\mathrm{d})$. In cases (b), (c), and (d) only the cross-frequency terms were used. As expected, the incoherent processors (a) and (d), gave similar smooth surfaces with a lower sidelobe structure for the crossfrequency processor. The coherent processors (b) and (c) also gave similar responses with a large number of very narrow peaks (up to only $1 \mathrm{~m}$ wide in range) that are due to a perfect alignment of the surfaces for all grid points. By formulating the matched-phase and the incoherent cross-frequency processors in terms of normal modes, it can be shown that the corresponding ambiguity surfaces are oscillating functions of the distance modulated by an amplitude factor that is the same in both processors. The peaky structure shown by the coherent processors results from a periodic phase alignment of the correlation terms at each pair of frequencies. At low SNR the coherent normalized processor (b), rapidly degrades due to the SNR limitation pointed out in (20). As explained above the matched-phase and the cross-frequency incoherent processors have analytically the same source detection performance with comparable peak-to-sidelobe ratios of $2.5 \mathrm{~dB}$ and $2.0 \mathrm{~dB}$, respectively. Note that for the coherent processors a subsampling of the ambiguity surface in range can hide the sidelobe structure. The detection performance of the 
(a)

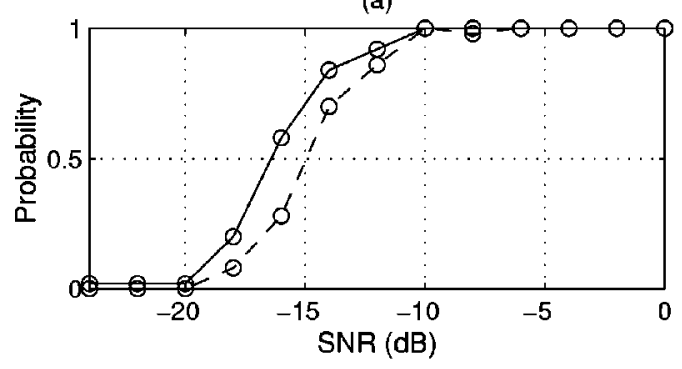

(c)

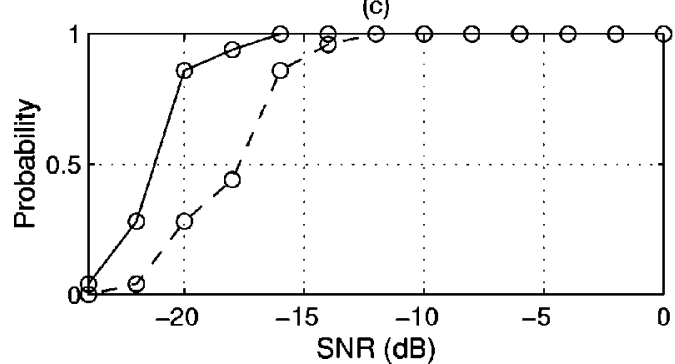

(b)

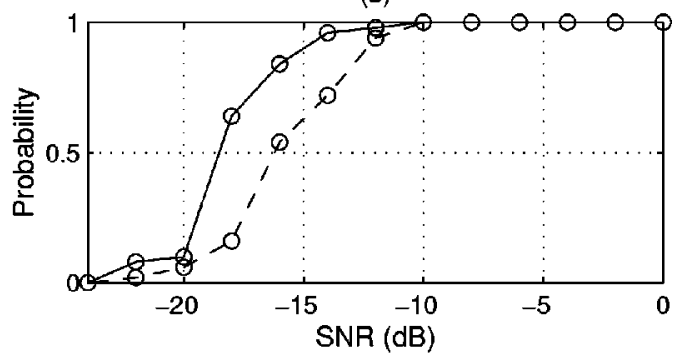

(d)

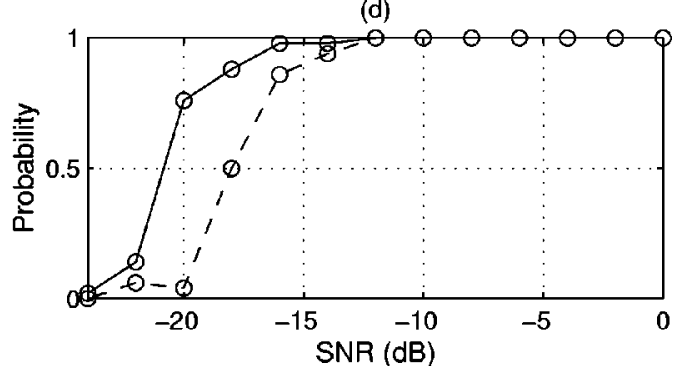

FIG. 2. Probability of correct source localization obtained on ADVENT'99 conditions simulated data for the broadband incoherent processor (dashed) and broadband cross-frequency processor (continuous) in the frequency band [500,600] Hz for the following number of frequencies: 4(a), 7(b), 16(c) and also 16 but in the band $[400,700] \mathrm{Hz}$.

cross-frequency incoherent processor is shown in Fig. 2 for the model without perturbation. This performance was measured in terms of probability of correct source localization by determining how often the peak appeared at the correct location in 50 realizations. The environment is always that of the ADVENT'99 experiment. The effect of increasing the number of frequencies within a relatively small frequency band of $100 \mathrm{~Hz}$ around $550 \mathrm{~Hz}$ is shown in plots (a) to (c) of Fig. 2, where the number of frequencies is 4,7 , and 16 , respectively. It can be noticed that the performance of the cross-frequency incoherent processor is always superior to that of the conventional (autofrequency) incoherent processor due to the higher number of frequencies involved and to the noise immunity, despite the inevitable decrease in channel structure power transmission at certain cross frequencies off the main diagonal. The number of ambiguity surfaces increases as $K$ for the incoherent processor and as $K \times(K$ $-1) / 2$ for the cross-frequency incoherent processor. That fact results in a steady increase of the difference in performance with the number of discrete frequencies from 4 to 7 and then to 16 . With 16 frequencies there is a gain in detection performance estimated to approximately $4 \mathrm{~dB}$ at useful detection probabilities. The result shown in plot (d) was obtained for a number of frequencies equal to 16 [the same as in plot (c)] but within a frequency band enlarged to $300 \mathrm{~Hz}$, always centered at $550 \mathrm{~Hz}$. The result is that there is a slight decrease of the performance of both processors, while that decrease is stronger for the incoherent cross frequency, thus there is a net loss of performance of the cross-frequency processor relative to the incoherent autofrequency processor. Other tests performed for relatively small number of closely spaced discrete frequencies clustered around center frequencies along the whole band gives better results than uniformly distributed frequencies in the same band.

In practice, with real data, these performance predictions obtained in simulation have to be balanced by the correlation of the perturbation factor across-frequency contributing to a net decrease of performance when enlarging the bandwidth around a given center frequency. That fact clearly favors the solution of using the proposed cross-frequency incoherent operating in closely spaced frequencies clustered at various frequencies in the useful band.

\section{A REAL DATA EXAMPLE}

The ADVENT'99 data set was used as to provide a real world example for the assertions made in the preceding sections. The ADVENT'99 experiment took place during the month of May of 1999, in a nearly range independent area on the Strait of Sicily, Italy. The approximate depth of the area was $80 \mathrm{~m}$ and the acoustic signals were transmitted from a bottom mounted sound source and received on a 31hydrophone vertical array. Various signal sequences at different frequencies and repetition rates were used. Also, the vertical array was successively located at ranges of approximately 2, 5, and $10 \mathrm{~km}$. A complete description of the experimental setup can be found in Siderius et al. ${ }^{21}$

\section{A. The perturbation factor}

In order to justify the perturbation factor distribution, a signal tone at $200 \mathrm{~Hz}$ was extracted from the time series recorded during 18 hours on a mid-water-depth hydrophone at $10 \mathrm{~km}$ range from the signal source. The signal-to-noise ratio is expected to be $>20 \mathrm{~dB}$ at that frequency, and the additive noise is assumed negligible compared to the signal term. Figure 3 shows the estimated pdf's based on the histograms obtained for the module-(a) and (c) —and for the phase-(b) and (d). In plots (a) and (b), module and phase pdf's are estimated using the normalization proposed in (6). It can be seen that the module is approximately Rayleigh distributed, with parameter $\lambda=1$ due to the normalization by $y_{0}$, while the phase is noncentered (also due to the normal- 
(a)

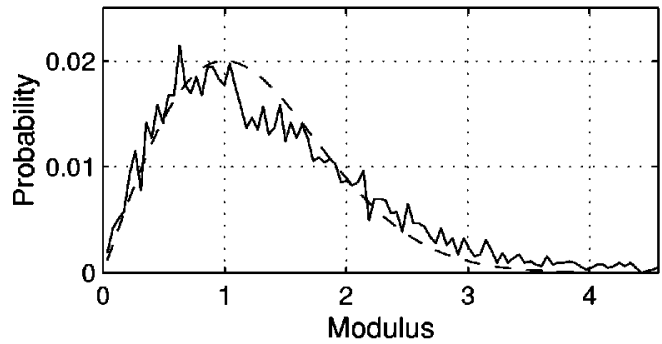

(c)

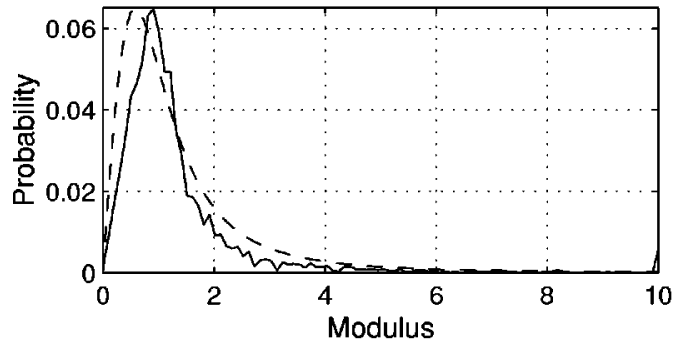

(b)

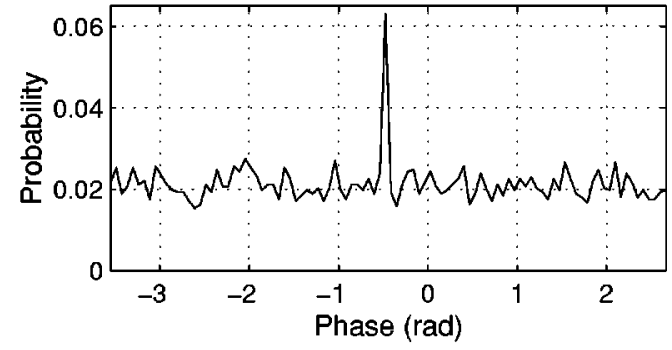

(d)

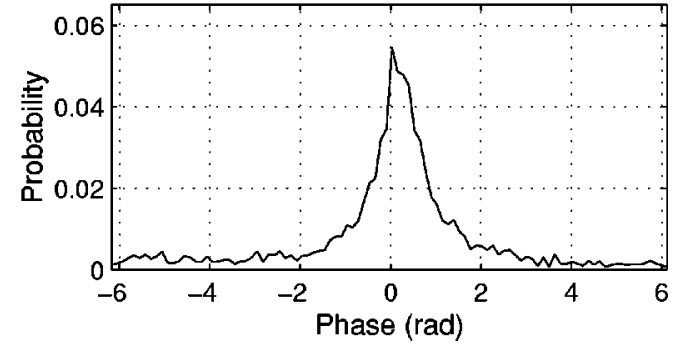

FIG. 3. Estimated probability density functions of signal perturbation factor from the ADVENT' 99 data set at $200 \mathrm{~Hz}$ : using first element normalization for the module (a) and phase (b); using a sliding window along time for module (c) and phase (d).

ization), almost uniform in $[-\pi, \pi]$ with an outstanding peak at $\phi=-0.5 \mathrm{rad}$ of unknown nature. Instead, the sliding normalization of (7), applied in the same data set, provides the results shown in plots (c) and (d) for the module and phase, respectively. The module_plot (c) — is in this case approximately distributed according to the approximate Cauchy given by (B2), while that of the phase-plot (d) - does not resembles to a triangular function as a result from the difference of two uniformly distributed random variables. The distribution is approximately symmetric in $[-2 \pi, 2 \pi]$, but has a much narrower peak than expected for a triangular shaped pdf. Due to the complicated form of the expression of the phase pdf in the noncentered case [Appendix A, Eq. (A14)], it is difficult to theoretically predict what could be the expected pdf for the phase random variable $\phi_{n}-\phi_{n-1}$. Some numerical simulations using expression (A14) and realistic values for $\sigma$ suggest that a bell-shaped centered pdf as that of Fig. 3(d) can most likely be obtained for $m_{a}>0$ and $m_{b}$ $\approx 0$. [Note that the empirical distribution of Fig. 3(d) is, according to the theory, the autocorrelation of two identical pdf's as that obtained in (A14)]. A similar behavior was verified on the ADVENT'99 data set at various frequencies in the interval $[200,1500] \mathrm{Hz}$, with however, an increasing broadening of the peak of the phase pdf with frequency. A broader pdf means a larger value for $\sigma$ which is a well known effect leading to highly variable phase shifts at high frequency making it difficult to accurately predict. This discussion brings a key question for broadband applications, that is to determine which is the degree of correlation of the signal across frequency.

Using the same ADVENT'99 data set along a wide frequency band $[200,1600] \mathrm{Hz}$, the correlation of the perturbation factor using the normalization (6) was estimated. The result is shown in Fig. 4 where a broad diagonal along the whole frequency band can be observed. Additional effects of frequency bandpass of the two transducers used to cover this wideband of frequencies can be seen on the artificially low levels of energy in the diagonal at about $800 \mathrm{~Hz}$, which is the overlap transit in frequency band.

In order to obtain a complete view of the received signal correlation along frequency one has to add the deterministic effect of the channel correlation. As an example, a scenario similar to that of the ADVENT'99 was simulated to compute the cross-array coherence of the acoustic channel across the frequency band of interest. Figure 5 shows the result of the expression

$$
\mathbf{C}_{h}\left(\omega_{i}, \omega_{j}\right)=\mathbf{h}^{H}\left(\omega_{i}\right) \mathbf{h}\left(\omega_{j}\right),
$$

for $\omega_{i}, \omega_{j} \in[200,1600] \mathrm{Hz}$. It can be easily noticed from that figure that the energy is not concentrated on a single diagonal but on a band of frequencies around that diagonal. The bandwidth varies with frequency and with sourcereceiver geometry (not shown), e.g., it tends to be larger at longer ranges due to stronger multipath. There is also a sig-

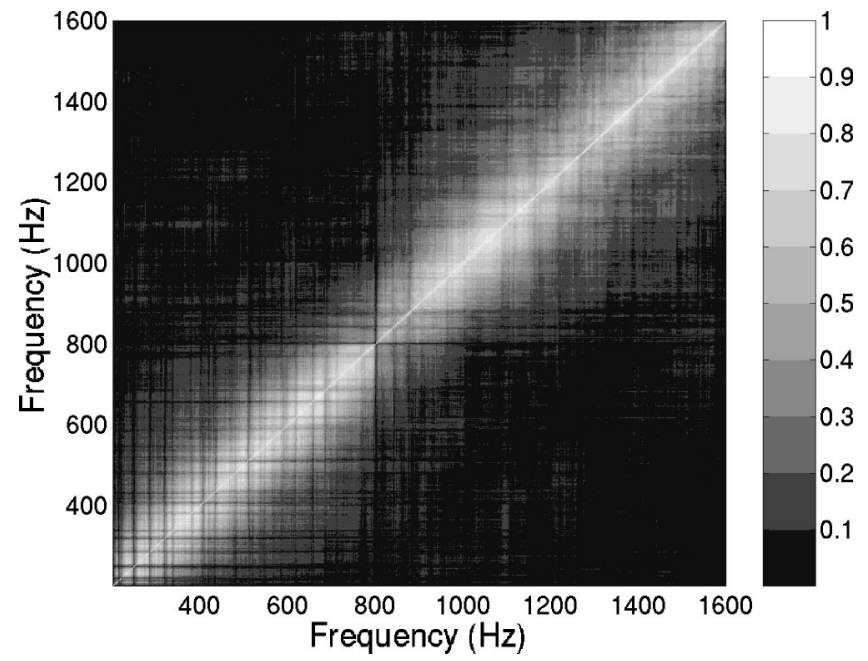

FIG. 4. Estimated correlation of normalized signal perturbation factor over the band 200-1600 Hz using the LFM data of the ADVENT'99 experiment at $5 \mathrm{~km}$ source-receiver range. 


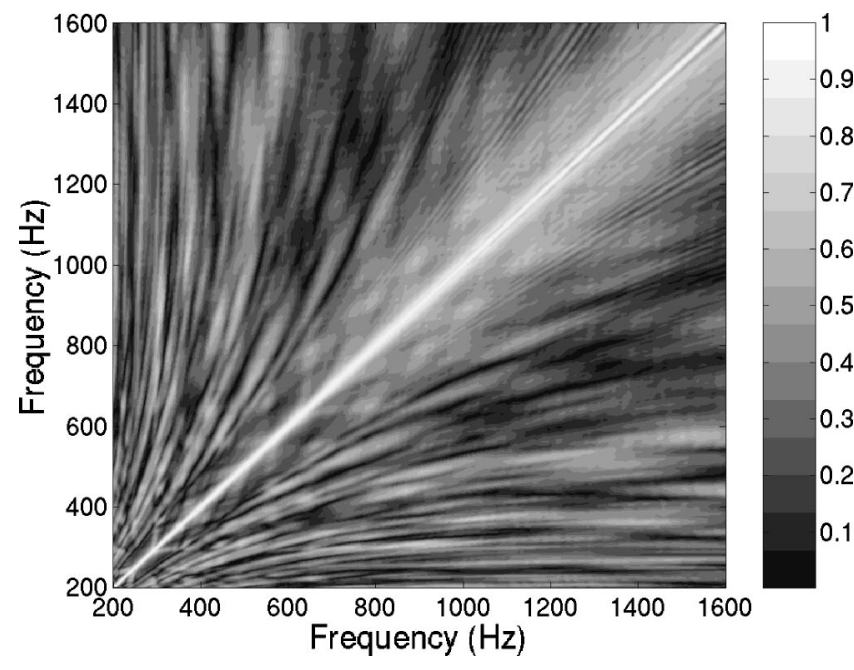

FIG. 5. Channel coherence of simulated acoustic field in the band 200-1600 $\mathrm{Hz}$ in the ADVENT'99 conditions with a source-receiver range of $5 \mathrm{~km}$.

nificant amount of energy well apart from the diagonal due to mode interference.

These two last Figs. 4 and 5 can be compared by means of a third figure that is the frequency correlation matrix of the signals received at $5 \mathrm{~km}$ range during the ADVENT'99 sea trial (Fig. 6). The first comment is that the resemblance between this figure and that obtained with simulated data is striking. It appears that the cross-frequency energy spread out of the diagonal is largely attenuated when compared with the synthetic data example, that is particularly true in the low frequency range but is also evident at high frequencies where the main diagonal lobe is narrower. An estimation of the effective $-3 \mathrm{~dB}$ bandwidth shows that at least $100 \mathrm{~Hz}$ are available throughout the analyzed frequency range between 200 and $1600 \mathrm{~Hz}$. Comparison of Figs. 5 and 6 should be done under the assumption that the latter contains information on the source spectrum level that might alter the result. Note that the values plotted in the last three figures were normalized, so there is no information on the relative levels of each term on the final observed signal.

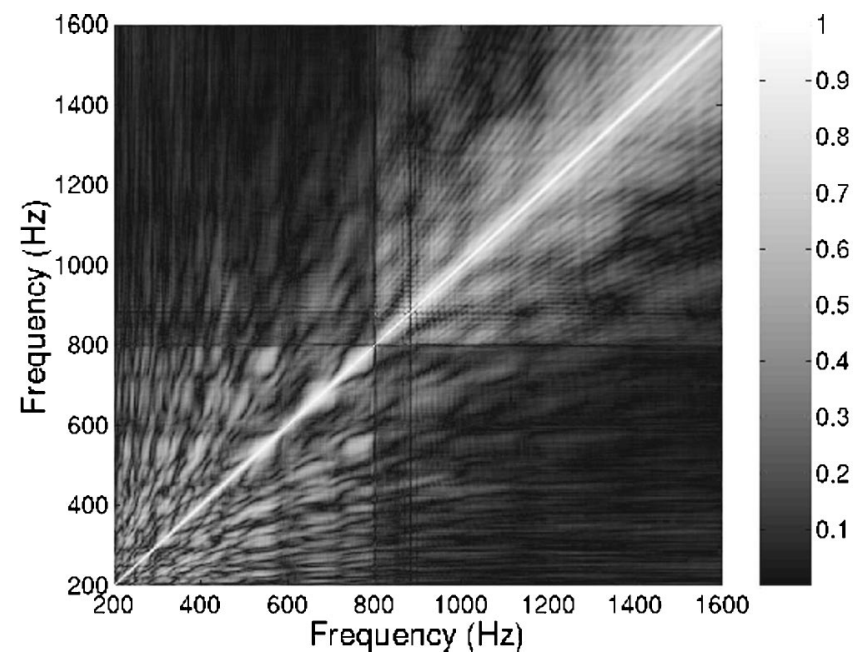

FIG. 6. Estimated correlation of received signal over the band 200-1600 Hz using the LFM data of the ADVENT' 99 experiment at $5 \mathrm{~km}$ source-receiver range.

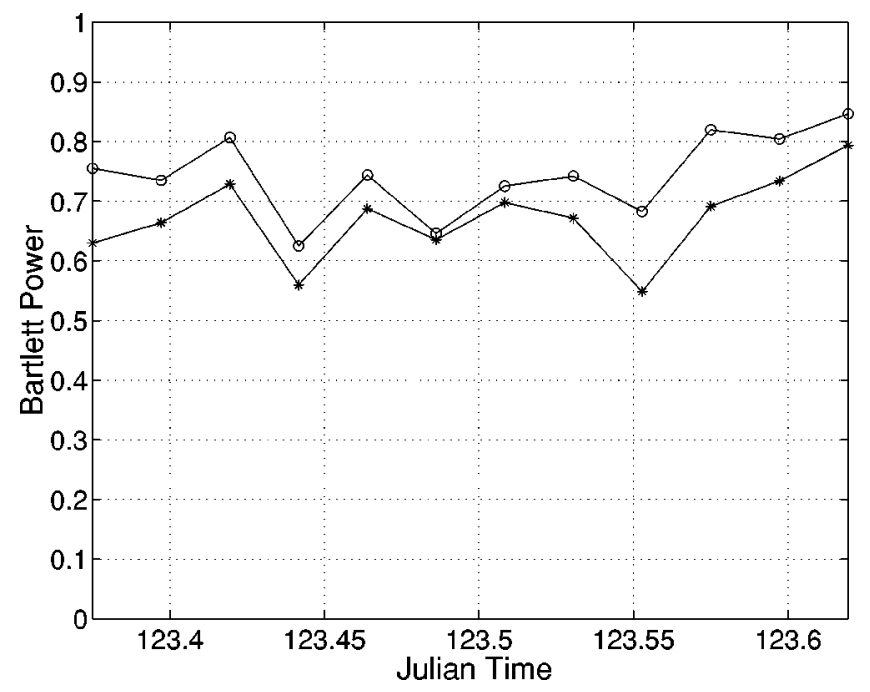

FIG. 7. Bartlett power for source localization using the cross-frequency incoherent processor in the $5 \mathrm{~km}$ range ADVENT'99 data set: $200 \mathrm{~Hz}$ frequency band clustered processing $(\mathrm{O})$ and $600 \mathrm{~Hz}$ wide band processing (*).

\section{B. Broadband MFP}

The results shown in the preceding section suggest that due to the limited correlation of the perturbation factor, cross-frequency broadband processors should preferably operate on relative narrow bands of 100 or $200 \mathrm{~Hz}$ than on wide frequency bands. In order to illustrate that point with a real data example, a series of vertical array observations at $5 \mathrm{~km}$ range and in the band $400-1000 \mathrm{~Hz}$ was drawn from the ADVENT'99 data set and processed with the proposed cross-frequency incoherent Bartlett processor for rangedepth source localization purpose. Figure 7 shows the Bartlett power results obtained during approximately five consecutive hours of data for two processing schemes: the seven tones at $100 \mathrm{~Hz}$ spacing between 400 and $1000 \mathrm{~Hz}$ were processed in a single frame $(*)$ and the same tones were processed in three groups of three frequencies each $(\mathrm{O})$ groups $(400,500,600),(600,700,800)$, and $(800,900,1000)$. The number of cross-frequency terms is 21 in the first scheme and nine in the second scheme. Despite that difference the Bartlett power, i.e., the value of the normalized peak in the final ambiguity surface at the correct source location, is always higher for the processing in the clustered band than in the wide band. The range-depth source localization performance was the same for both processors. The grouping of frequencies in a limited band acts as an automatic scheme to exclude the correlation terms that yield worst SNR at the processor's output caused by low cross correlation of the signal components.

\section{CONCLUSION}

For many years, underwater acoustic signal processing was devoted to the detection and/or localization of narrowband or broadband random sources using a multisensor horizontal array. Localization here meant bearing estimation, which was the main scope of a wide, yet powerful, suite of techniques. That situation has dramatically changed with the wide spread of physical model codes being able to predict the acoustic channel propagation characteristics at various 
ranges and depths and in different environmental conditions with practical relevance. These are the generically called matched-field (MF) techniques, that are used not only for detecting and localizing submerged targets but also, and more importantly, to probe the ocean (ocean tomography) and the seafloor (geoacoustic inversion). From a purely signal processing point of view, the problem has lost most of its interest since the knowledge of an image of the received signal limits the range of (optimum) methods to the wellknown matched filter. However, numerous tests with real data have shown that physical models, at least in their present form, can not account for acoustic channel fluctuations between the source and the receiver(s).

This paper approaches the problem of modeling the received signal as a mixture of a deterministic structure, that can be predicted by a suitable acoustic model, and a random perturbation factor that is supposed to be space invariant (within the physical sensor array limits) and time variant. Estimation of that perturbation factor on the ADVENT'99 data set has shown that its amplitude was approximately Rayleigh distributed but its phase did not follow a uniform distribution as it is assumed in many texts. Those distributions were apparently frequency invariant with, however, a consistent variance increase for the random perturbation phase term. It was also shown, based on the same real data set, that a band of frequencies extending to $100 \mathrm{~Hz}$ can be safely assumed to contain a significant channel and random perturbation cross-frequency signal correlation.

Making use of that data model allowed for derivations of optimum broadband MF processors, according to the various assumptions on the signal and perturbation factor correlation across frequency. The uncorrelated perturbation assumption led to the well-known incoherent broadband processor. The often used unweighted processor was shown to be optimum only on the flat source spectrum case. Other coherent broadband processors proposed in the literature are shown to provide either suboptimum performance in real noisy situations or to have serious limitations in terms of the number of frequencies processed in a reasonable computation time. An alternative incoherent algorithm is proposed that is shown to have the same detection performance as the matched-phase coherent processor. That processor-the incoherent crossfrequency processor-is able to process any number of frequencies with only a slightly larger computation time than that of the incoherent processor with however, the advantage of using the asymptotically noise-free cross-frequency terms and without making any use of the source spectrum. In that sense the proposed incoherent cross-frequency processor can be compared with that developed by Westwood, ${ }^{15}$ since neither used the source spectrum knowledge with, however, one main difference that is that the former uses cross-frequency terms while the latter only used autofrequencies. Finally, a simple simulated test on realistic conditions, illustrated the detection performance of the proposed cross-frequency incoherent processor when compared with the autofrequency incoherent processor for a well chosen frequency band relative to the band of coherence of the underwater channel. It was concluded that the cross-frequency processor always outperformed the autofrequency processor clearly showing that it was advantageous to chose clustered sets of closely spaced discrete frequencies instead of an equivalent number of uniformly distributed frequencies along the whole band.

\section{ACKNOWLEDGMENTS}

The authors would like to thank Jürgen Sellschopp, Martin Siderius and Peter Nielsen responsible for the ADVENT'99 experiment design and data collection, and the SACLANT Undersea Research Center for providing the data of the ADVENT'99 experiment. This work was partially financed by Fundação para a Ciência e a Tecnologia-FCT, Portugal, under Contract No. ATOMS,PDCTM/P/MAR/ 15296/1999.

\section{APPENDIX A: ENVELOPE AND PHASE DISTRIBUTIONS}

Let $\alpha=a+j b$ be a random variable such that

$$
a: N\left(0, \sigma^{2}\right) \text { and } b: N\left(0, \sigma^{2}\right),
$$

where $a$ and $b$ are uncorrelated, in which case it is well known that the polar notation $\alpha=|\alpha| \exp (j \phi)$ implies that

$$
\begin{aligned}
& |\alpha|: R\left[\sigma \sqrt{\frac{\pi}{2}}, \sigma^{2}\left(2-\frac{\pi}{2}\right)\right], \\
& \Phi: U_{-\pi, \pi}\left(0, \frac{\pi}{\sqrt{3}}\right),
\end{aligned}
$$

where $R$ and $U$ designate Rayleigh and Uniform distributions, respectively. The question is to determine the distribution of $V=|\alpha|$ and $\Phi$ when $a$ and $b$ are not zero mean. So, let us assume that

$$
a: N\left(m_{a}, \sigma^{2}\right), \quad b: N\left(m_{b}, \sigma^{2}\right),
$$

with joint probability density function (pdf)

$$
p_{A, B}(a, b)=\frac{1}{2 \pi \sigma^{2}} \exp \left[-\frac{\left(a-m_{a}\right)^{2}+\left(b-m_{b}\right)^{2}}{2 \sigma^{2}}\right] .
$$

It is known that the square module $Y=A^{2}+B^{2}$ follows a noncentral chi-square distribution $\chi^{2}(s)$ with the noncentrality parameter $s^{2}=m_{a}^{2}+m_{b}^{2}$. The pdf of $Y$ is given by

$$
p_{Y}(y)=\frac{1}{2 \sigma^{2}} \exp \left(-\frac{y+s^{2}}{2 \sigma^{2}}\right) I_{0}\left(\frac{\sqrt{y} s}{\sigma^{2}}\right), \quad y \geqslant 0
$$

where $I_{0}$ is the zeroth-order modified Bessel function of first kind. Thus a simple change of variable $R=\sqrt{Y}$ gives us the pdf of $R$ as

$$
p_{R}(r)=p_{Y}\left(r^{2}\right)|J|,
$$

where $J=2 r$ is the Jacobian of the transformation giving

$$
p_{R}(r)=\frac{r}{\sigma^{2}} \exp \left(-\frac{r^{2}+s^{2}}{2 \sigma^{2}}\right) I_{0}\left(\frac{r s}{\sigma^{2}}\right), \quad r \geqslant 0,
$$

which represents a Rice distribution with parameter $s^{2}=a^{2}$ $+b^{2}$.

For the phase $\phi$ the calculation is more elaborated and the result is not easy to interpret. Let us first make the transformation

$$
\left\{\begin{array} { l } 
{ V ^ { 2 } = A ^ { 2 } + B ^ { 2 } } \\
{ \Phi = \operatorname { a r c t a n } ( B / A ) }
\end{array} \Leftrightarrow \left\{\begin{array}{l}
A=V \cos \Phi, \\
B=V \sin \Phi
\end{array}\right.\right.
$$


with the Jacobian, $|J|=v$, thus the joint pdf of the new variables $(V, \Phi)$ is

$$
\begin{aligned}
& p_{V, \Phi}(v, \phi) \\
& \quad=p_{A, B}(a, b)|J| \\
& \quad=\frac{v}{2 \pi \sigma^{2}} \exp \left[-\frac{\left(v \cos \phi-m_{a}\right)^{2}+\left(v \sin \phi-m_{b}\right)^{2}}{2 \sigma^{2}}\right] .
\end{aligned}
$$

The marginal distribution can be obtained as

$$
p_{\Phi}(\phi)=\int_{0}^{\infty} p_{V, \Phi}(v, \phi) d v
$$

The first step to solve the integral obtained by replacing (A7) in (A8) is to develop the sum of squares in the exponent to get (only for the exponent)

$$
-\frac{1}{2 \sigma^{2}}\left[v^{2}-2 v\left(m_{a} \cos \phi+m_{b} \sin \phi\right)+m_{a}^{2}+m_{b}^{2}\right]
$$

which can be made a square of the sum, by subtracting and adding the term $\left(m_{a} \sin \phi-m_{b} \cos \phi\right)^{2}$ which gives for the pdf,

$$
\begin{aligned}
p_{\Phi}(\phi)= & \frac{1}{2 \pi \sigma^{2}} e^{-\left(m_{a} \sin \phi+m_{b} \cos \phi\right)^{2} / 2 \sigma^{2}} \\
& \times \int_{0}^{\infty} v \exp \left\{-\frac{\left[v-\left(m_{a} \cos \phi+m_{b} \sin \phi\right)\right]^{2}}{2 \sigma^{2}}\right\} d v .
\end{aligned}
$$

Performing a change of variable $z=v-\left(m_{a} \cos \phi\right.$ $+m_{b} \sin \phi$ ) reduces to

$$
\begin{aligned}
p_{\Phi}(\phi)= & \frac{1}{2 \pi \sigma^{2}} e^{-\left(m_{a} \sin \phi-m_{b} \cos \phi\right)^{2} / 2 \sigma^{2}} \\
& \times \int_{-\left(m_{a} \cos \phi+m_{b} \sin \phi\right)}^{\infty} z e^{-z^{2} / 2 \sigma^{2}} d z+\cdots \\
& +\frac{m_{a} \cos \phi+m_{b} \sin \phi}{2 \pi \sigma^{2}} e^{-\left(m_{a} \sin \phi-m_{b} \cos \phi\right)^{2} / 2 \sigma^{2}} \\
& \times \int_{-\left(m_{a} \cos \phi+m_{b} \sin \phi\right)}^{\infty} e^{-z^{2} / 2 \sigma^{2}} d z .
\end{aligned}
$$

The first integral equates to

$$
\exp \left[-\frac{\left(m_{a} \cos \phi+m_{b} \sin \phi\right)^{2}}{2 \sigma^{2}}\right]
$$

that gives by replacement in (A11) and by knowing that the second integral is even, allows for changing the sign of the integration bounds

$$
\begin{aligned}
p_{\Phi}(\phi)= & \frac{\exp \left(-\frac{m_{a}^{2}+m_{b}^{2}}{2 \sigma^{2}}\right)}{2 \pi \sigma^{2}}+\cdots+\frac{m_{a} \cos \phi+m_{b} \sin \phi}{2 \pi \sigma^{2}} \\
& \times e^{-\left(m_{a} \sin \phi-m_{b} \cos \phi\right)^{2} / 2 \sigma^{2}} \\
& \times \int_{-\infty}^{m_{a} \cos \phi+m_{b} \sin \phi} e^{-z^{2} / 2 \sigma^{2}} d z .
\end{aligned}
$$

Now, a small change of variable $\lambda=z / \sigma$ allows to view this last integral as the distribution function of a standard normally distributed random variable as

$$
\begin{aligned}
p_{\Phi}(\phi)= & \frac{\exp \left(-\frac{m_{a}^{2}+m_{b}^{2}}{2 \sigma^{2}}\right)}{2 \pi \sigma^{2}}+\cdots+\frac{m_{a} \cos \phi+m_{b} \sin \phi}{2 \pi \sigma} \\
& \times e^{-\left(m_{a} \sin \phi-m_{b} \cos \phi\right)^{2} / 2 \sigma^{2}} \\
& \times \int_{-\infty}^{\left(m_{a} \cos \phi+m_{b} \sin \phi\right) / \sigma} e^{-\lambda^{2} / 2} d \lambda
\end{aligned}
$$

It is not possible to continue any further knowing the difficulties to calculate the integral in the second term. Available approximate expressions exist for large $m_{a} \cos \phi$ $+m_{b} \sin \phi / \sigma$ but that assumption does not makes much sense for the problem at hand.

\section{APPENDIX B: ESTIMATING THE RANDOM PERTURBATION FACTOR DISTRIBUTION}

Let $\alpha_{n}$ and $\alpha_{n-1}$ be two independent Rayleigh distributed random variables with pdf's,

$$
p_{\alpha}=\frac{\alpha}{\lambda} e^{-\alpha^{2} / 2 \lambda^{2}}, \quad \alpha \geqslant 0 .
$$

The random variable $Z$ defined as $Z=\alpha_{n} / \alpha_{n-1}$ can be shown to follow a pdf as

$$
p_{Z}(z)=\frac{2 \lambda_{n}^{2}}{\lambda_{n-1}^{2}} \frac{z}{\left(z^{2}+\lambda_{n}^{2} / \lambda_{n-1}^{2}\right)^{2}}, \quad z \geqslant 0 .
$$

Separately, if $\Phi_{n}$ and $\Phi_{n-1}$ are two independent Uniformly distributed random variables in $[-\pi, \pi]$, then it can be easily demonstrated that the pdf of the random variable $\Delta \Phi=\Phi_{n}-\Phi_{n-1}$ is given by the correlation between the pdf's of the two random variables $\Phi_{n}$ and $\Phi_{n-1}$, i.e.,

$$
p_{\Delta \Phi}(\Delta \phi)=\int_{-\infty}^{\infty} p_{\Phi_{n}}(\Delta \phi+\tau) p_{\Phi_{n-1}}(\tau) d \tau,
$$

which, can be easily evaluated for Uniform distributions as $p_{\Delta \Phi}(\Delta \phi)=\left\{\begin{array}{l}\frac{1}{8 \pi^{2}}(2 \pi-\Delta \phi), \quad-2 \pi \leqslant \Delta \phi \leqslant 2 \pi, \\ 0, \quad \text { otherwise. }\end{array}\right.$

${ }^{1}$ H. P. Bucker, "Use of calculated sound fields and matched-detection to locate sound source in shallow water," J. Acoust. Soc. Am. 59, 368-373 (1976)

${ }^{2}$ M. J. Hinich, "Maximum-likelihood signal processing for a vertical array," J. Acoust. Soc. Am. 54, 499-503 (1973).

${ }^{3}$ A. B. Baggeroer, W. A. Kuperman, and P. N. Mikhalevsky, "An overview 
of matched field methods in ocean acoustics," IEEE J. Ocean. Eng. 18, 401-424 (1993).

${ }^{4}$ A. B. Baggeroer, W. A. Kuperman, and H. Schmidt, "Matched field processing: Source localization in correlated noise as an optimum parameter estimation problem," J. Acoust. Soc. Am. 83, 571-587 (1988).

${ }^{5} \mathrm{~A}$. Tolstoy, "Computational aspects of matched field processing in underwater acoustics," in Computational Acoustics, edited by D. Lee, A. Cakmak, and R. Vichnevetsky (North-Holland, Amsterdam, 1990), Vol. 3, pp. 303-310.

${ }^{6} \mathrm{Z}$.-H. Michalopoulou, "Matched-field processing for broadband source localization,” IEEE J. Ocean. Eng. 21, 384-392 (1996).

${ }^{7}$ Z.-H. Michalopoulou, "Source tracking in the Hudson Canyon experiment," J. Comput. Acoust. 4, 371-383 (1996).

${ }^{8}$ S. P. Czenszak and J. L. Krolik, "Robust wideband matched-field processing with a short vertical array," J. Acoust. Soc. Am. 101, 749-759 (1997).

${ }^{9}$ G. J. Orris, M. Nicholas, and J. S. Perkins, "The matched-phase coherent multi-frequency matched field processor," J. Acoust. Soc. Am. 107, 25632575 (2000).

${ }^{10}$ C. S. Clay, "Optimum time domain signal transmission and source location in a waveguide,” J. Acoust. Soc. Am. 81, 660-664 (1987).

${ }^{11} \mathrm{~S}$. Li and C. S. Clay, "Optimum time domain signal transmission and source location in a waveguide: Experiments in an ideal wedge waveguide," J. Acoust. Soc. Am. 82, 1409-1417 (1987).

${ }^{12}$ L. N. Frazer and P. I. Pecholcs, "Single-hydrophone localization," J. Acoust. Soc. Am. 88, 995-1002 (1992).
${ }^{13}$ J. H. Miller and C. S. Chiu, "Localization of the sources of short duration acoustic signals," J. Acoust. Soc. Am. 92, 2997-2999 (1992).

${ }^{14}$ D. P. Knobles and S. K. Mitchell, "Broadband localization by matched fields in range and bearing in shallow water," J. Acoust. Soc. Am. 96, 1813-1820 (1994).

${ }^{15}$ E. K. Westwood, "Broadband matched-field source localization," J. Acoust. Soc. Am. 91, 2777-2789 (1992).

${ }^{16}$ R. K. Brienzo and W. S. Hodgkiss, "Broadband matched-field processing," J. Acoust. Soc. Am. 94, 2821-2831 (1993).

${ }^{17}$ I. Tolstoy and C. S. Clay, Ocean Acoustics: Theory and Experiments in Underwater Sound (AIP, New York, 1966).

${ }^{18} \mathrm{~W}$. B. Davenport, Jr. and W. L. Root, An Introduction to the Theory of Random Signals and Noise (IEEE Press, New York, 1987).

${ }^{19}$ Z.-H. Michalopoulou, "Robust multi-tonal matched-field inversion: A coherent approach," J. Acoust. Soc. Am. 104, 163-170 (1998).

${ }^{20}$ C. M. Ferla, M. B. Porter, and F. B. Jensen, "C-SNAP: Coupled SACLANTCEN normal mode propagation loss model," Memorandum SM-274, SACLANTCEN Undersea Research Center, La Spezia, Italy, 1993.

${ }^{21}$ M. Siderius, P. L. Nielsen, J. Sellschopp, M. Snellen, and D. G. Simons, "Experimental study of geo-acoustic inversion uncertainty due to ocean sound-speed fluctuations," J. Acoust. Soc. Am. 110, 769-781 (2001). 